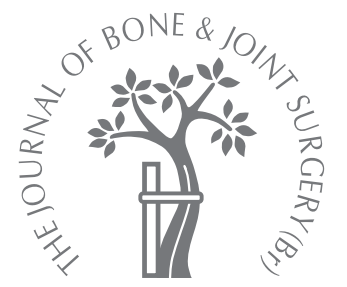

- CASE REPORT

\title{
Medial dislocation of the medial meniscus
}

\author{
S. K. L. Chan, \\ C. A. Robb, \\ T. Singh, \\ S. Chugh \\ From New Cross \\ Hospital, \\ Wolverhampton, \\ England
}

\footnotetext{
S. K. L. Chan, MRCS, Orthopaedic ST3

- C. A. Robb, MRCS (Ed), Orthopaedic Registrar

T. Singh, MRCS, Orthopaedic Fellow

- S. Chugh, FRCS (Orth), Consultant Orthopaedic Surgeon

New Cross Hospital Wednesfield Road, Wolverhampton, West Midlands, WV10 0QP, UK.

Correspondence should be sent to Dr S. K. L. Chan; email:sammychan98@hotmail. com
}

(C)2010 British Editorial Society of Bone and Joint Surgery doi:10.1302/0301-620X.92B1. $22298 \$ 2.00$

$J$ Bone Joint Surg $[\mathrm{Br}]$ 2010;92-B:155-7. Received 13 January 2009; Accepted after revision 10 August 2009

\begin{abstract}
We present the first reported case of symptomatic medial dislocation of the medial meniscus in a patient who had no previous history of trauma and who had an otherwise normal knee. The treatment of instability of the medial meniscus is controversial and studies have indicated that certain individuals without a firm meniscal bony insertion may be predisposed to meniscal dislocation. In our patient, the meniscal instability interfered with daily activities. Operative stabilisation by reconstruction of the meniscotibial ligaments cured the symptoms.
\end{abstract}

Dislocation or subluxation of the medial meniscus is rare. Sir Robert Jones first acknowledged subluxation of the anterior horn in $1925^{1}$ in his discussion of Bristow's paper. ${ }^{2}$ It is still an uncommon condition despite the use of improved imaging techniques.

There is no documented instance of medial dislocation of the medial meniscus and the only comparable cases have either involved anterior dislocation of the anterior horn ${ }^{3}$ or posterior dislocation secondary to trauma. ${ }^{4}$ We present a case of symptomatic medial dislocation of the medial meniscus and discuss options for treatment.

\section{Case report}

In 2001, a 14-year-old boy was referred with a two-year history of pain and crepitus on the medial side of the right knee. There was no history of trauma, locking or 'giving way' and he was otherwise fit and well. On examination there was a mobile cherry-sized swelling which appeared on hyperflexion with an audible click and disappeared in extension. Plain radiographs were normal. A provisional diagnosis of a meniscal tear or cyst was made. Arthroscopy showed a hypermobile medial meniscus subluxating medially off a normal tibial plateau. No operative treatment was undertaken and he was referred for physiotherapy.

On review after six months, he felt that his knee was weak and giving way. There had been progression of the pain particularly on kneeling. This was limiting his daily activities and he was having difficulty in walking long distances and running.
He was referred back to us in July 2002. On examination there was full active and passive movement. The knee had normal alignment, there was no patellofemoral or patellar tendon tenderness and no tenderness or laxity of the collateral ligaments. McMurray's test reproduced a clunk medially in flexion and Lachmann's test showed grade-1 laxity with a definite endpoint. There remained a mobile lump which appeared with a loud click on the medial aspect of the knee in full flexion (Fig. 1). On MRI the medial meniscus appeared normal, but there was an increased signal in the posterior horn which was reported as 'innocent'.

He underwent a further examination under anaesthesia and an arthroscopy. The former revealed a marginally lax medial collateral ligament (MCL), but was otherwise normal. Arthroscopy showed an intact medial meniscus and meniscosynovial junction. There was no chondral wear in the medial tibial or femoral condyles. However, the anterior and middle thirds of the meniscus were thought to be hypermobile and overhanging the medial tibial plateau. This was thought to be a normal anatomical variant, presumably as a result of deficient meniscotibial ligaments. Arthroscopic examination of the rest of the knee showed no abnormality.

Post-operatively, he recovered well and returned to reasonable function, including football, although the lump was still reproducible. However, after 15 months, the symptoms of pain and locking returned and persisted, despite physiotherapy. 


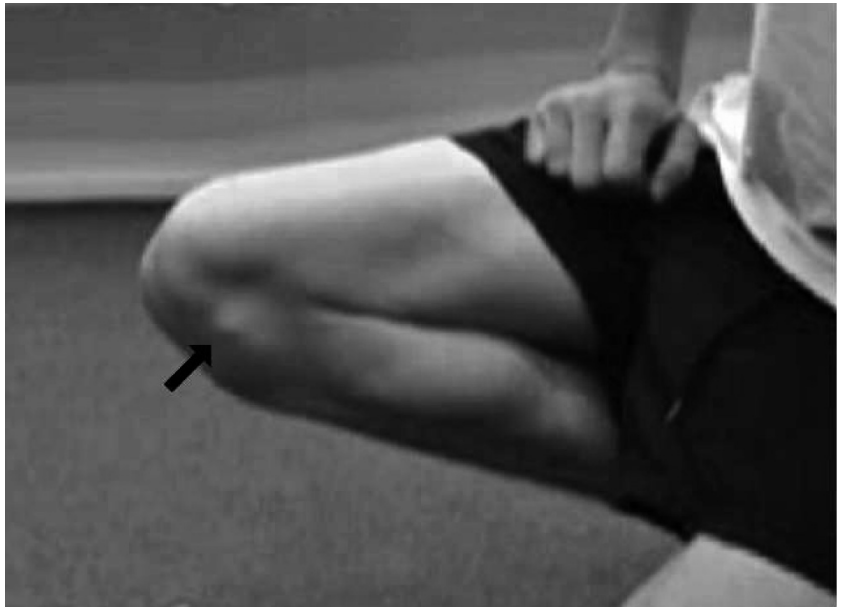

Fig. 1

Photograph showing meniscal dislocation (arrow) in hyperflexion.

He was referred back to us and dynamic ultrasonography showed a hypermobile meniscus dislocating as before in hyperflexion (Fig. 2). He was advised to have operative stabilisation of the meniscus.

This was performed through a medial approach. A preliminary arthroscopy excluded new pathology and confirmed the dislocation. A longitudinal incision was made over the site of the dislocation and the MCL was incised in the line of its fibres. The loose capsular tissue was opened and a burr was used to freshen the bone margin around the medial tibial plateau to promote adhesion. The meniscotibial ligaments were reconstructed using three Mitek anchors (DePuy, Mitek Inc, Raynham, Massachusetts) sutured to the meniscal margins. The loose capsular tissue was reefed and double-breasted.

Post-operatively, a plaster-of-Paris cylinder was applied for four weeks with mobilisation and weight-bearing as tolerated. Flexion beyond $90^{\circ}$ was restricted for a further two weeks before full movement was allowed. He was advised to avoid squatting for three months.

At follow-up at 12 months he had regained full movement and returned to normal activities. He has had no further episodes of dislocation.

\section{Discussion}

Anatomically, the medial meniscus is described as a semilunar structure which is less mobile than its lateral counterpart because of soft tissue and bony attachments. ${ }^{5}$ The meniscotibial ligaments are continuous with the joint capsule, attaching the inferior edges of the fibrocartilaginous meniscus to the periphery of the tibial plateau. Posteriorly, the medial meniscus is anchored by the transverse ligament, which also stabilises the lateral meniscus, and is fixed to the posterior intercondylar area between the attachments of the lateral meniscus and the posterior cruciate ligament. Medially, it is attached to the deep aspect of the MCL. Anteriorly, it is firmly attached to the intercondylar area of the tibia a few millimetres

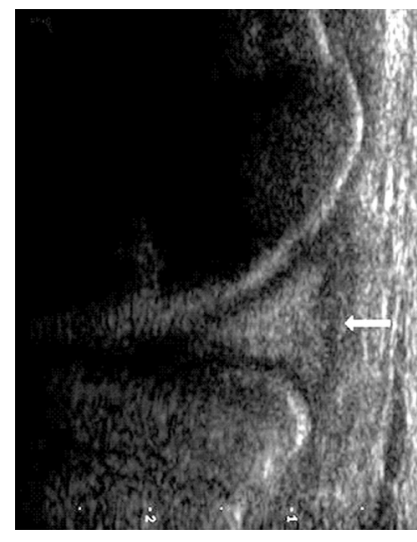

Fig. 2a

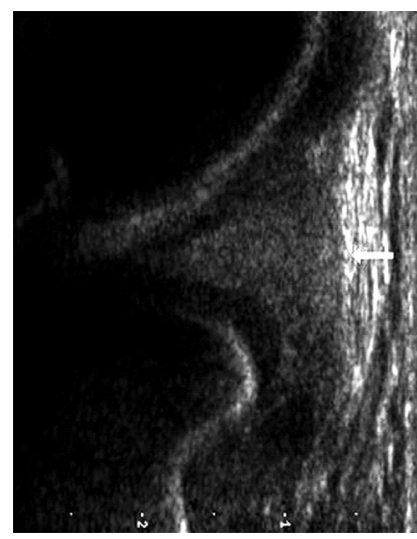

Fig. 2b
Dynamic ultrasonography of the knee showing a) the meniscus in the joint on standing and b) the meniscus extruded medially when squatting.

below the periphery of the articular surface, together with the meniscomeniscal and the meniscocruciate ligaments. ${ }^{6}$

Berlet and Fowler ${ }^{6}$ defined this further in a cadaver study. They reported four types of anterior location of the tibial insertion of the medial meniscus. In type I (59\%) the insertion was on the flat intercondylar region of the tibial plateau. In type II $(24 \%)$ it was on the downward slope from the medial articular plateau to the intercondylar region. In type III (15\%) it was on the anterior slope of the tibial plateau and in type IV $(3 \%)$ there was no firm bony insertion. They suggested that type III and IV may not be able to resist peripheral extrusion of the loaded meniscus. Our patient had insufficiency of both anterior and medial attachments.

In isolation, deficient anterior attachments seem to be a normal variant. Pinar et $\mathrm{al}^{3}$ believed that re-attachment was not justified since it was an incidental finding. However, they described mainly meniscal dislocations in the context of trauma and found that operative treatment of the associated pathology in the knee resulted in the resolution of symptoms.

Clancy, Keene and Goletz ${ }^{7}$ advocated arthroscopic excision of the anterior horn of the medial meniscus in symptomatic knees since there may be corresponding erosion of the cartilage of the medial femoral condyle. However, in an isolated meniscal dislocation, excision of the meniscus is difficult to justify.

More recently, Matsui and $\mathrm{Oishi}^{8}$ showed that anterior meniscal dislocations could be symptomatic and advocated stabilisation of the anterior horn using a variety of techniques including staples, wires and suture anchors. In 13 patients they found a marked improvement in the Japanese Orthopaedic Association meniscal score following fixation at a mean follow-up of 20 months.

Although the above studies do not directly relate to the circumferential instability described in our case, conclusions can be extrapolated with regard to the treatment of meniscal instability. 
We believe that our patient had deficient anterior and medial attachments of the medial meniscus and minor or repetitive trauma could have further destabilised the meniscus, particularly if the meniscotibial ligaments were detached. Since the dislocation was occuring in hyperflexion, it was likely that the medial femoral condyle reduced the space and increased the pressure in the medial compartment sufficiently to cause the meniscus to dislocate medially. This was shown well by dynamic ultrasonography.

No benefits in any form have been received or will be received from a commerical party related directly or indirectly to the subject of this article.

\section{References}

1. Jones R. Internal derangement of the knee: general discussion. J Bone Joint Surg 1925;7:439-43.

2. Bristow WR. Internal derangement of the knee joint. J Bone Joint Surg 1925;7:413-17.

3. Pinar H, Akseki D, Bozkurt M, Karaoglan O. Dislocating anterior horn of the medial meniscus. Arthoscopy 1998;14: 246-9.

4. Pagnani MJ, Cooper DE, Warren RF. Extrusion of the medial meniscus. Arthroscopy 1991;7:297-300.

5. Gray. Gray's anatomy: the anatomical basis of medicine and surgery. Standring S, Ellis $\mathrm{H}$ Berkovitz BKB, eds. Thirty-ninth ed. Edinburgh: Elsevier, Churchill Livingstone, 2005.

6. Berlet GC, Fowler PJ. The anterior horn of the medial meniscus: an anatomic study of its insertion. Am J Sports Med 1998;26:540-3.

7. Clancy WG, Keene JS, Goletz TH. Symptomatic dislocation of the anterior horn of the medial meniscus. Am J Sports Med 1984;12:57-64.

8. Matsui Y, Oishi Y. Arthroscopic stabilization of the symptomatic unstable anterior segments of the medial meniscus of the knee. J Bone Joint Surg [Br]2002;84-B (Supp III):264. 